\title{
Quantum Dot Sensitized Whisperonic Solar Cells-Improving Efficiency Through Whispering Gallery Modes
}

\author{
Athrey Cholasettyhalli Dakshinamurthy, Tapan Kumar Das, P. Ilaiyaraja and \\ Chandran Sudakar*
}

Multifunctional Materials Laboratory, Department of Physics, Indian Institute of Technology Madras, Chennai, India

OPEN ACCESS

Edited by:

Peter Reiss,

Commissariat à l'Energie Atomique et aux Energies Alternatives

(CEA), France

Reviewed by:

Silvia Soria,

Nello Carrara Institute of Applied

Physics (IFAC), Italy

Jialiang Wang,

University of Wisconsin-Madison,

United States

*Correspondence: Chandran Sudakar csudakar@iitm.ac.in

Specialty section:

This article was submitted to

Energy Materials,

a section of the journal

Frontiers in Materials

Received: 21 August 2019

Accepted: 22 October 2019

Published: 15 November 2019

Citation:

Cholasettyhalli Dakshinamurthy A, Das TK, Ilaiyaraja P and Sudakar C

(2019) Quantum Dot Sensitized

Whisperonic Solar Cells-Improving

Efficiency Through Whispering Gallery

Modes. Front. Mater. 6:282.

doi: 10.3389/fmats.2019.00282
Environmental deterioration and depletion in conventional energy resources greatly demand the need for photovoltaic devices, which use solar radiation to meet future energy demands. Efficient light management plays a pivotal role in improving the performance of photovoltaic devices. Various avenues have been explored to address light management in solar cells. Employing whispering gallery mode (WGM) microresonators in solar cell device is one such strategy. Using resonating structures for light scattering is recently gaining momentum as they exhibit great potential to enhance the efficiency through light trapping. Functional material-based microresonators further provide an added advantage as they combine inherent optical resonance with the material properties suitable for photovoltaics like efficient charge separation and transport in one platform. "Whisperonic solar cell" is a broadly classified device in which resonating cavities are used in the cell architecture to effectively scatter the light, resulting in enhanced light absorption and thus efficiency. Recent studies reveal that WGM-enabled optical microcavities can effectively get coupled to the light absorber in a sensitized solar cell (SSC) and improve the performance of SSC significantly. In this short review, we briefly present the idea of enhancing the efficiency of solar cell using WGMs. Several case studies available from the literature for realizing the concept of WGM for light trapping are highlighted. Particular focus is given to the quantum dot sensitized whisperonic solar cells. The concept is much more universal and will be useful in both thin-film and sensitized solar cells.

Keywords: whispering gallery mode, microresonator, DSSC, QDSSC, perovskite

\section{INTRODUCTION}

Solar radiation, which is green, clean, and abundant, is a vast resource for renewable energy technologies. Recent changes in climatic conditions and increased global energy demands have prompted researchers to develop technologies and device efficient methods to get useful form of energy from such renewable resources. Quantum dot sensitized solar cells (QDSSCs), which belong to the third-generation photovoltaic devices, have drawn enormous attention in recent years because of the possibility of boosting their efficiencies beyond the theoretical limit proposed by Shockley-Quiesser (Nozik, 2002). In a typical QDSSC device, quantum dots (QDs) are the active light absorbing material (Kamat, 2008). QDs are three-dimensionally confined, optically active nanocrystals whose dimensions are less than or comparable with their Bohr exciton radius (Bera et al., 2010). Early report on QDSSCs in a dye-sensitized solar cell (DSSC) configuration, using 
indium phosphide (InP) QD as the active light-absorbing sensitizer, appeared in 1998 (Zaban et al., 1998). QDs possess exceptional optical properties, such as multiple exciton generation, large absorption coefficient, excellent photostability, size-dependent bandgap tunability, large dipole moments for enhanced charge separation, and many more (Nozik, 2002; Kamat, 2008; Rühle et al., 2010).

In spite of possessing exceptional optical properties, the performance of QDSSCs in terms of efficiency is sub-average when compared to silicon-based devices (Sharma et al., 2015). Extensive research is being carried out to improve the performance of these solar cells. Complex processes are involved in QDSSCs, which include light absorption, charge separation, and charge transport across multiple heterojunctions. Thus, optimization of different components of the cell including choice of QDs, its size and quality (Hetsch et al., 2011; Jara et al., 2014), electrolyte, type of photoanode, counter electrode (Jun et al., 2013), etc. have become an intense focus of research. Few other strategies are being investigated for bettering the performance of cells. These include surface passivation (Zhao et al., 2012), molecular linker assistance (Pernik et al., 2011), device architecture (Zhu et al., 2013), light trapping for enhanced absorption (Kim and Yong, 2013), adopting whispering gallery resonators in the photoanode (Das et al., 2018a), etc.

The challenge that prevails even today is to perfect various avenues available to achieve high efficiency. Among several of the listed parameters, designing photoanode is of paramount importance (Sharma et al., 2016). Most of the loss in solar cell device results from the ineffective charge carrier collection at photoanode. These arise from poor connectivity by the nanoparticles of photoanode, poor charge separation and transport in the photoanode layer, ineffective loading of the sensitizer on the photoanode, and non-directional transport of charge carriers with significant recombination (Mora-Seró et al., 2009; Tachan et al., 2013; Prasad and Pathan, 2016). Inefficient light absorption by QDs and non-optimal utilization of light entering a device is the most decisive one. A large fraction of light that enters the solar cell gets lost or does not get converted into excitonic pairs (Shen et al., 2015). To overcome such hindrance, researchers have modified, for example, the photoanode in a Grätzel-type solar cell (O'Regan and Grätzel, 1991). One notable version is the plasmonic solar cell (Catchpole and Polman, 2008). Another concept recently gaining momentum is the use of whispering gallery modes (WGMs) to effectively scatter the light (Grandidier et al., 2011; Das et al., 2018a; Wang et al., 2018a). The latter has led to a new type of solar cell broadly classified as "whisperonic solar cell" (Das et al., 2018a). The photoanode in these devices is modified so as to exhibit optical whispering gallery resonances while it exhibits the rest of all the characteristics required for the functioning of SSCs. Optical whispering galleries employed in other solar cells have been shown to improvise the net efficiency of the device. This short review provides the basic background of optical WGMs and highlights from recent works the efforts made to enhance the efficiency of different types of solar cells. A detailed summary of various studies, with specific focus on the QD and perovskite SSCs, that has integrated the
WGM emitting microresonators in the solar cell devices is given (also refer to Table 1).

\section{WHISPERING GALLERY RESONANCE}

Resonant phenomena in optical cavities are analogous to the acoustic WGMs first explained by Rayleigh $(1910,1914)$. WGMs occur in optical cavities with precise geometric properties possessing closed concave interface guided by means of repeated reflections (Foreman et al., 2015). Mie theory provides a theoretical framework for elastic (scattering) interaction between plane waves and spherical objects (van de Hulst, 1946). The first experimental observation of WGM optical resonances was demonstrated with a spherical sample of $\mathrm{CaF}_{2}: \mathrm{Sm}^{++}$ (Garrett et al., 1961). Micron-sized spheres of $\mathrm{SiO}_{2}, \mathrm{TiO}_{2}$, $\mathrm{Si}_{2} \mathrm{~N}_{3}, \mathrm{SiC}$, polymethyl methacrylate, polystyrene, etc. have been used as optical cavities in optoelectronic sensing applications (Venkatakrishnarao et al., 2018). Total internal reflection at the cavity interface, where the refractive index of the cavity is greater than outside ambient, leads to the confinement and also helps in the sustainment of WGMs (Foreman et al., 2015). The negligible radiative losses, absorption, scattering, and material dispersion leads to stronger confinement of WGM and highest $Q$ factors are achievable. Light with wavelength " $\lambda$ " resonates in spherical cavity by the propagation and circulation of scattered light in a ring-like path. For a spherical cavity of radius " $a$ " and refractive index " $n(\lambda)$ ", under the constructive interference condition, WGMs are guided in the resonator. A simple approximation for the constructive resonance to occur in a spherical cavity is that the circumference should be equivalent to an integral multiple $(m)$ of the wavelength of light inside the sphere, i.e., $2 \pi a=m \lambda^{\prime}$, where $\lambda^{\prime}=\frac{\lambda}{n(\lambda)}$. A schematic diagram depicting the formation of WGM in a spherical resonator is given in Figure 1A. It is much more complicated to get a precise spectrum of WGM for a given geometry and composition of resonator (Foreman et al., 2015). While a general analytic solution describing the modal structures does not exist, for spherical symmetry resonators, using exact analytical methods, solutions for scattering of light have been proposed (Mie, 1908; Debye, 1909). Direct observation of WGM is not feasible as the energy is trapped in the cavity. However, WGM can be observed from the photoluminescence spectra emitted from the sphere itself. Emission of light with modulated wavelength and intensity also occurs due to the surface losses when the resonator is continuously excited. Such spectra are analogous to the scattering efficiency $\left(Q_{\text {sca }}\right)$ of those resonators (Chang and Campillo, 1996). A representative photoluminescence spectrum obtained from the $\mathrm{TiO}_{2}$ microsphere clearly revealing the formation of WGM is shown in Figure 1B. The excitation of WGM also becomes evident when the resonator is coated with fluorescent material like dye or QD. Due to the increase in the local density of states, fluorescence gets enhanced many folds (Foreman et al., 2015). An example of such an enhanced fluorescence resulting from the CdSe QD loaded on the $\mathrm{TiO}_{2}$ microsphere is shown in Figure 1C. Thus, such modulated light sources are found to be effective means of enhancing 
TABLE 1 | Solar cells using whispering gallery resonating structures to enhance power conversion efficiency.

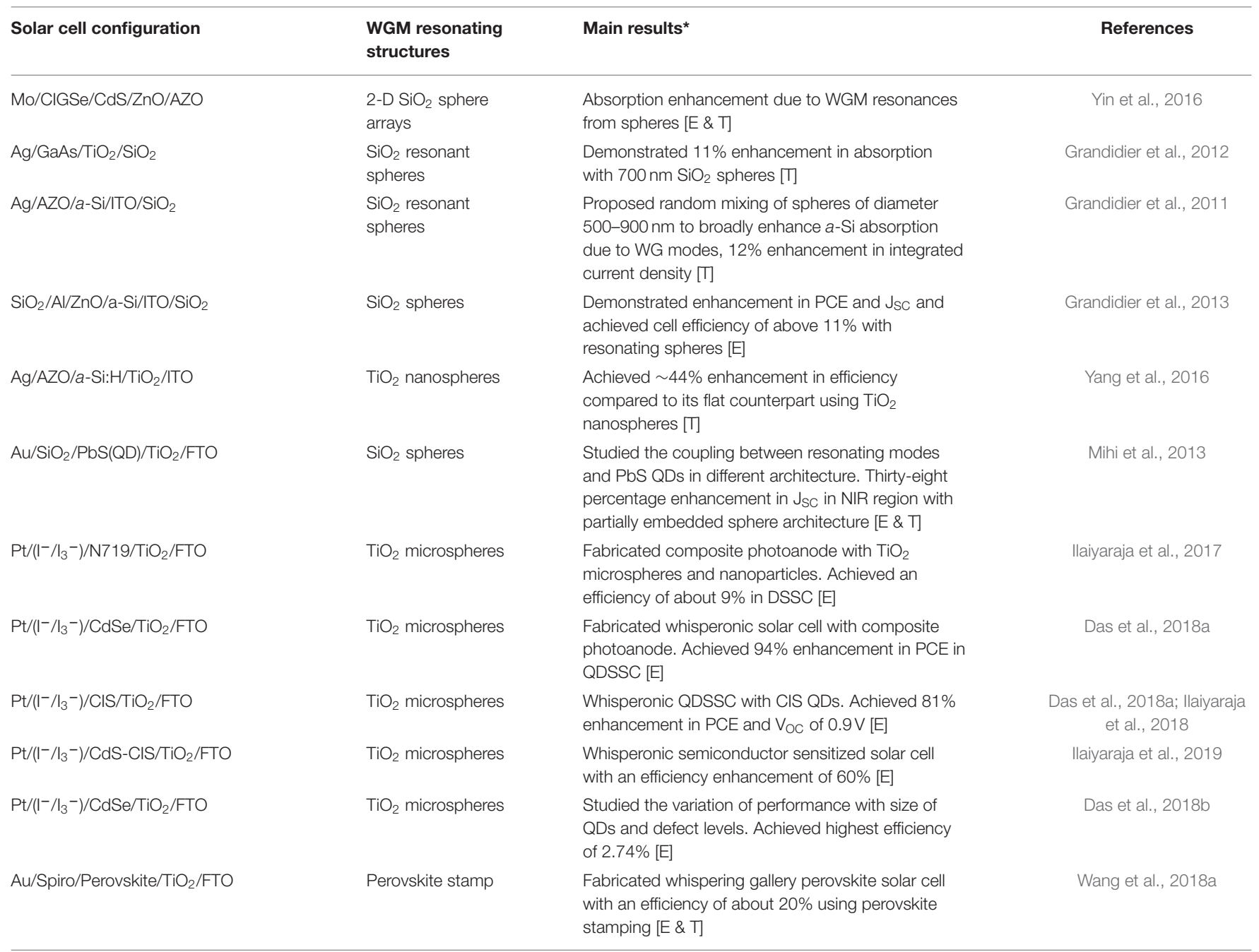

${ }^{*}[E]$ refers to experimental studies and [T] refers to theoretical/numerical simulations.

the absorption and conversion of light to energy in solar cell devices.

\section{APPLICATION OF WGM IN SOLAR CELLS WGM in Thin-Film Solar Cells}

Silicon solar cells suffer with poor absorption characteristics of silicon, which demands thick film deposition to absorb the sunlight effectively (Wang et al., 2010). Various strategies have been explored to address this issue, which include antireflection coating of dielectric thin films (Zhao et al., 1995) and surface patterning with nanocones (Zhu et al., 2009) and nanopyramids (Mavrokefalos et al., 2012), which gave tangible results. However, the increased surface area resulting from the patterned nanostructures cause an undesired recombination loss and thus decreases the open-circuit voltage, in turn limiting the power conversion efficiency (Ha et al., 2018). Significant enhancement in absorptivity without compromising the opencircuit voltage has been reported by texturing the surface with cellulose fibers, which gradually changes the refractive index from air to the active material. Such alteration has resulted in $24 \%$ enhancement in the power conversion efficiency of GaAs solar cells (Ha et al., 2014). Nanostructured solar cells that belong to a novel class of photovoltaics have the potential to reach the power conversion efficiency up to $42 \%$, which is because of the improvement in open circuit voltage arising from the built-in optical concentration that leads to higher carrier densities (Xu et al., 2015). Recently, other promising approaches are gaining attention to enhance the light absorption in solar cells. These include exciting and coupling the optical resonance modes using spherical resonators (Yao et al., 2012; Yin et al., 2013). The incident light is excited into resonant modes using spherical resonators and optically coupled to the absorbing medium. WGMs being mainly the surface modes, photonic whispering gallery excitation ultimately leads to enhanced light absorption within the high index active material of solar cells (Ha et al., 2018). A complete mechanism behind the efficiency enhancement through WGM is yet to be understood clearly. 


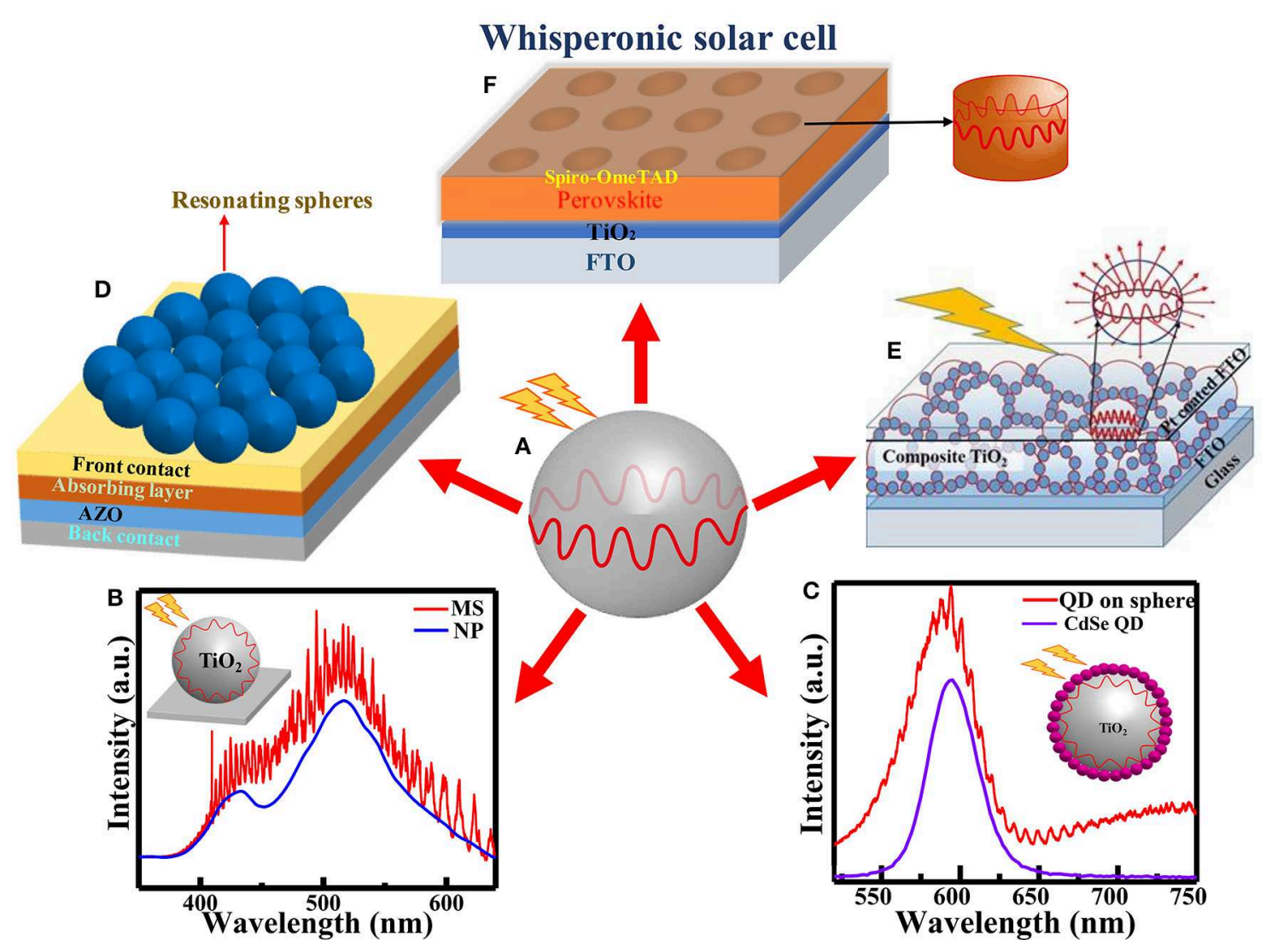

FIGURE 1 | (A) Schematic of WGM in a spherical microcavity. (B) PL spectra of isolated $\mathrm{TiO}_{2}$ microsphere and its nanoparticle counterpart (adapted from Das et al., 2018a, with permission from American Chemical Society). (C) PL spectra of bare CdSe QD and QD loaded on $\mathrm{TiO}_{2}$ microsphere (adapted from Das et al., 2018a, with permission from American Chemical Society). Application of WGM resonators in different solar cell architectures: (D) a-Si solar cell with close packed dielectric resonant nanospheres (adapted from Grandidier et al., 2011, with permission from John Wiley and Sons). (E) Schematic of whisperonic QDSSC (adapted from Das et al., 2018b, with permission from Springer Nature). (F) Schematic of whispering gallery perovskite solar cell (adapted from Wang et al., 2018a, Copyright Elsevier).

From finite-difference time-domain (FDTD) simulations, it has been shown that power absorbed is directly proportional to the intensity of electric fields (Grandidier, 2012). Thus, the most commonly cited reasoning is the increase in electric field intensities arising from whispering gallery resonances at the interface between resonator array and the photosensitive material (Ha et al., 2018). Yin et al. have shown the enhancement in the absorption of silicon by decorating optical whispering gallery resonators on the surface of Si solar cells (Yin et al., 2013). Further, Grandidier et al. theoretically showed that WGM in the spheres can be coupled into particular modes of the solar cell, thus significantly enhancing the efficiency (Grandidier et al., 2011). They have investigated several absorber configurations using a periodic array of resonant silica microspheres and demonstrated significant enhancement of light absorption in amorphous silicon solar cells. Figure 1D depicts a schematic of such $a$-Si solar cell decorated with close packed dielectric resonant nanospheres on top of the cell (Grandidier et al., 2011). As the WGMs vary with the size of resonators, randomly mixing spheres with a diameter ranging from 500 to $900 \mathrm{~nm}$ was proposed as a way to broadly enhance the
$a$-Si absorption. Since $a$-Si has poor absorption characteristics for $\lambda>600 \mathrm{~nm}$, this approach was expected to improve the efficiency largely (Grandidier et al., 2011). Light trapping due to microcavities could enable reduction in the thickness of an absorber layer resulting in electrical improvement and cost reduction as experimentally demonstrated by Grandidier et al. Efficiency over $11 \%$ in thin-film silicon solar cells using resonant dielectric structures was demonstrated (Grandidier et al., 2013). Alternatively, silicon nanoshell structures are shown to exhibit good confinement of WGM, because of high refractive index contrast between air/substrate and the nanocrystalline silicon (Yao et al., 2012). They also maintain a low $Q$ factor, which facilitates the coupling (Yao et al., 2012). Twenty-fold reduction in the film thickness was achieved without compromising the absorption when spherical silicon shells were used in comparison to a thick planar silicon solar cells (Yao et al., 2012). Thus, WGM resonators with a low $Q$ factor have high absorption, low frequency selectivity, and high coupling efficiency (Yao et al., 2012; Hua et al., 2013). Yang et al. also proposed a novel approach to enhance the light-trapping capability and the light conversion efficiency 
of the ultrathin $a$-Si:H solar cells (Yang et al., 2016). In this work, $\mathrm{TiO}_{2}$ nanosphere arrays along with ITO coating were made on top of the $a-\mathrm{Si}: \mathrm{H}$ layer. Such cells were shown to exhibit $\sim 44 \%$ enhancement in efficiency compared to the cell without the resonant spheres. Apart from the studies on Si-based p-n junction solar cells, Yin et al. have explained the absorption enhancement in ultrathin CIGSe solar cells (Yin et al., 2016). Closely packed $\mathrm{SiO}_{2}$ sphere arrays were used in their studies. It has been demonstrated that the WGM and Mie scattering, which dominate in the larger spheres, can enhance the light absorption for CIGSe solar cells. Smaller spheres on the other hand are also proven to enhance the absorption as these spheres form an anti-reflection layer (Yin et al., 2016). Dielectric nanospheres decorated on the solar cells can gradually change the refractive index from air to the high index active material, thus favoring absorption enhancement (Ha et al., 2018). Recently, Ha et al. have demonstrated nearly $20 \%$ enhancement in absorptivity and photocurrent using silicon dioxide $\left(\mathrm{SiO}_{2}\right)$ nanosphere arrays on a gallium arsenide (GaAs) solar cell. The absorptivity enhancement observed over the entire visible spectrum is attributed to an antireflective effect arising from thin-film interference. In addition, the narrowband absorptivity enhancements over the various wavelengths are evidenced, which is attributed to the enhanced electric field intensities arising from whispering gallery resonances at the interface between nanosphere array and GaAs substrate. Photocurrent enhancement has resulted from the improved number of photo-generated electron-hole pairs due to broadband enhancement in absorptivity. Thus, total enhancement was attributed to the combined effects of thin-film interference and whispering gallery-like resonances within nanosphere arrays (Ha et al., 2018).

\section{WGM to Boost the Efficiency in DSSCs/QDSSCs}

QDs, unlike silicon, possess exceptional optical properties, such as large optical absorption coefficient (Yu et al., 2003). However, their performance still does not match with that of thin-film solar cell devices. If the morphology of $\mathrm{TiO}_{2}$ nanoparticulate photoanodes, which suits SSC well due to their high surface area and catalytic property, is tailored for superior light scattering, it will make solar cells much more promising for energy conversion applications (Wang et al., 2004). Early research in the field of QD-embedded microcavities mainly explored lasing and sensing applications (Shopova et al., 2004; Zhi et al., 2013). Beier et al. have developed models to understand the WGMs in QDembedded polystyrene microspheres (Beier et al., 2010). The interface between the microsphere and QD was demonstrated to serve as local light source, thus increasing the sensitivity of microspheres (Beier et al., 2010). QD-coupled WGM-based refractive index sensors have been demonstrated by Zhi et al., wherein a thin layer of fluorescent silicon QDs on the surface of silica microsphere was used (Zhi et al., 2012). Aneesh et al. have studied the effect of WGM resonances on the photoluminescence properties of CdSe QDs loaded on silica microsphere and observed an enhancement in $Q$ factors of WGM upon coating with CdSe QDs (Veluthandath and Bisht, 2015). However, in the field of QDSSCs, the applicability of WGM resonators is rarely explored until recently. Major factors that limit the power conversion efficiency in SSCs include the energy loss due to charge recombination and ineffective light management. The latter was addressed to a large extent by adopting different architectures of photoanodes (Liao et al., 2012). Several studies on DSSC devices used a microsphere-nanoparticulate bilayer or a composite $\mathrm{TiO}_{2}$ photoanode (Dwivedi et al., 2013; Qadir et al., 2015). A DSSC device made up of $\mathrm{TiO}_{2}$ mesoporous nanoparticles and microsphere composites as photoanodes exhibited an efficiency $(\eta)$ increase from 7.1 to $8.9 \%$ (Ilaiyaraja et al., 2017) and 7.13 to 7.94\% (Qadir et al., 2015). These studies clearly revealed the advantage of microspherical light scattering particles in the photoanode. Effective increase of $\eta$ by $25 \%$ and $11.3 \%$, respectively, compared to the nanoparticulate counterpart in these works is very promising. The composite structure proposed by Ilaiyaraja et al. possesses properties of high light scattering, higher surface area, and low interfacial resistance, which are desired for efficient electron generation and transport (Ilaiyaraja et al., 2017). Later, it was shown that a significant increase in efficiency, in fact, arises from the increased absorption by the dye, mainly modulated by the WGM arising from the microspheres (Das et al., 2018a). Similar enhancement was shown to prevail in QDSSC and semiconductor sensitized solar cell (SCSSC) devices (Das et al., 2018a; Ilaiyaraja et al., 2019). Extensive studies carried out by fabricating QDSSC devices using CdSe QDs and CuInS 2 QDs are reported (Das et al., 2018a; Ilaiyaraja et al., 2018). In these studies, composite mesoporous photoanodes with microspherical $\mathrm{TiO}_{2}$ light scattering particles were used. Such devices are generically termed "quantum dot sensitized whisperonic solar cell (QDSWSC)" as the whispering gallery resonant modes from $\mathrm{TiO}_{2}$ microspheres (Figure 1B) exhibited strong coupling with QDs as demonstrated by photoluminescence spectroscopy (Figure 1C). Such strong coupling persists even after the coating of the sensitizer on the surface of smooth microspheres in the photoanode (Ilaiyaraja et al., 2019). Interestingly, the QD emission spectra from sensitized photoanodes exhibited significant blue shift compared to bare QD emission (Figure 1C). The presence of resonating modes and the blue shift in the sensitizer emission peak in the PL spectrum of QD-loaded photoanodes strongly indicate an efficient coupling between scattered light from microsphere and $\mathrm{QD} /$ dye absorption. The photoconversion efficiency of a CdSebased whisperonic solar cell is found to be $2.7 \%$, which is nearly $94 \%$ enhancement compared to the mesoporous nanoparticle photoanode. The schematic of a QDSWSC is shown in Figure 1E. The whisperonic solar cells made with $\mathrm{CuInS}_{2}$ QDs also show a similar trend with the highest PCE of 3.8\%, when the WGM exhibiting microspheres form a component in the photoanode. This amounts to $81 \%$ enhancement when compared to the photoanode without these light trapping resonators. Such a definite enhancement in the efficiency when large-sized spheres are employed in the photoanode certainly suggests that the resonant scattering due to WGM plays a vital role (Das et al., 2018a). Mie scattering leads to light trapping within the spheres, which create resonant modes with a wide range of wavelengths 
suitable for efficient energy conversion. In addition, the enhanced intensity of certain modes leads to the availability of large flux for the excitation process. These processes eventually lead to efficient light absorption and charge generation followed by the charge separation. The performance of whisperonic solar cells when tested with CdSe QDs of different sizes revealed that defect influenced photovoltaic performances. Surface disorders, which are prominent in smaller-sized QDs, appear to underperform, specifically in the process involving charge generation and extraction. With an increase in size, the surface defects go down and thus the PV performance increases. It is observed that a 5fold increase in the efficiency of QDSWSC is achieved with a QD of $4.7 \mathrm{~nm}$ size in comparison with 2.5-nm-sized QDs (Das et al., 2018b). An unprecedented high $\mathrm{V}_{\mathrm{OC}}$ of $926 \mathrm{mV}$ is also evidenced when QDSSCs with $\mathrm{CuInS}_{2}$ QDs are made using a microsphere-nanoparticle composite photoanode (Ilaiyaraja et al., 2018). Low interfacial resistance and long electron lifetimes are observed in these composite photoanodes. The WGMenabled photoanodes are also shown to improve the thinfilm SSCs (Ilaiyaraja et al., 2019). Optical whispering galleryenabled $\mathrm{CdS}-\mathrm{CuInS} \mathrm{S}_{2}$ thin-film whisperonic solar cells showed a champion cell efficiency of $4.3 \%$ with an average efficiency of $3.2 \%$ tested over several cells. However, the same thin-filmbased device without WGM-enabled photoanode showed an average efficiency of $1.9 \%$. This observed efficiency is the highest for CdS-CIS thin-film SSCs made using $\mathrm{I}^{-} / \mathrm{I}_{3}^{-}$electrolyte. This remarkable increase in average efficiency $(\sim 60 \%)$ is attributed to increased photon absorption by the sensitizer films because of the presence of WGM scattering prevailing in smooth microspheres (Ilaiyaraja et al., 2019).

\section{Whispering Gallery Architecture in Perovskite Solar Cells}

Recent reports reveal that the perovskite material-based thinfilm solar cells have revolutionized the photovoltaic technology with their remarkable efficiencies (Zhou et al., 2014). While theoretical efficiency is predicted to be $31 \%$ (Sha et al., 2015), Yang et al., have fabricated a perovskite solar cell with a certified efficiency of above 22\% (Yang et al., 2017). Very recently, Ling et al. have developed a solar cell with cesium-based perovskite QDs as sensitizers with a record efficiency of above 14\% (Ling et al., 2019). Inspired by these device performances, researchers have been modifying the device architectures for better light harvesting. One such interesting work reported by Wang et al. constructed the active perovskite layer such that it exhibits whispering gallery resonance modes. This has been enabled through a simple imprinted process with a robust microstructural stamp. A schematic of such a device with whispering gallery resonator pits in perovskite solar cells is shown in Figure 1F (Wang et al., 2018a). They observed that these whispering gallery structured perovskite films could achieve light trapping by optical feedback and gradually absorb the reflected light. This method is also shown to suppress recombination and efficiently accelerate electron-hole separation assisted by the arrayed column structure. A remarkable power conversion efficiency of $19.80 \%$ has been achieved with whispering gallery architecture, which was $29.4 \%$ higher than that of a nonwhispering gallery structured device (Wang et al., 2018a). In another report, Wang et al. developed a facile strategy to introduce a large area grating structure into the active perovskite layer of a solar cell by utilizing commercial optical discs and to achieve high photovoltaic performance. The constructed diffraction grating on the active layer realizes nanophotonic light trapping through diffraction and suppresses carrier recombination effectively. The observed power conversion efficiency for this device is $19.71 \%$ with a photocurrent density of $23.11 \mathrm{~mA} / \mathrm{cm}^{2}$ (Wang et al., 2018b).

\section{CONCLUSION}

In this review, we have summarized recent works addressing efficient light management in solar cells using WGMs. Mesoporous $\mathrm{TiO}_{2}$ nanoparticulates are well-suited for SSC applications as photoanodes. However, significant loss in photoconversion efficiency occurs due to the inefficient light absorption. Various schemes are being employed to address this, including suppression of specular reflection through antireflection coating (Tran et al., 2016) and surface texturing to increase diffuse reflection (Tang et al., 2014). Evolution of plasmonic solar cells paved a way to address the issue of light trapping to a certain extent (Catchpole and Polman, 2008). Alternatively, the concept of light trapping using whispering gallery microresonators is found to be an efficient way to improve light harvesting (Kang et al., 2013). WGMenabled light scattering was shown to improve the efficiency in Si solar cells (Grandidier et al., 2011). Photovoltaic device configuration with a modified Grätzel cell architecture with the inclusion of whispering gallery resonating structures in the photoanode was proposed as a whisperonic solar cell (Das et al., 2018a). Such structure is shown to enhance the efficiency of DSSC and QDSSC devices. Carving out such resonating structures in the absorbing layers itself has shown to be beneficial for improving the efficiency. Such device structures were shown to increase the efficiency of perovskite solar cells recently (Wang et al., 2018a). We envisage, therefore, that the WGM structures integrated in the solar cell device will enable the efficiency enhancement significantly and such solar cells can be universally classified as "Whisperonic solar cells."

\section{Future Scope}

Unique spectral properties of WGMs, including narrow line width and long lifetime, make whispering gallery resonators useful for numerous practical applications. We foresee ample scope to implement the WGMs in solar cells. Though spherical cavity is an extensively studied microstructure, other forms, such as hexagonal and microtubular cavities will also be favorable for solar cells. Much more detailed understanding is still required to effectively utilize these optically active and resonating cavities in solar cell devices. Also, WGMs are very much dependent on the geometry and refractive index of the cavity material. Exploring different materials, 
which have compatible optical properties for photovoltaic research, are also essential. Another specific research focus could be on understanding the interaction of WGM with the photosensitive material. Enhancement in the absorption characteristics of photosensitive materials and increased lifetime of excited carriers with minimization of charge recombination is yet another avenue to be explored in these devices. Finally, how far beyond the Shockley-Quiesser limit one can push the efficiency up theoretically and the actual outcome from the experiments using whisperonic solar cells remain to be answered.

\section{REFERENCES}

Beier, H. T., Coté, G. L., and Meissner, K. E. (2010). Modeling whispering gallery modes in quantum dot embedded polystyrene microspheres. J. Opt. Soc. Am. B 27, 536-543. doi: 10.1364/JOSAB.27.000536

Bera, D., Qian, L., Tseng, T.-K., and Holloway, P. H. (2010). Quantum dots and their multimodal applications: a review. Materials (Basel) 3, 2260-2345. doi: 10.3390/ma3042260

Catchpole, K. R., and Polman, A. (2008). Plasmonic solar cells. Opt. Express 16, 21793-21800. doi: 10.1364/OE.16.021793

Chang, R. K., and Campillo, A. J. (1996). Optical Processes in Microcavities. Singapore: World Scientific Publishing Company.

Das, T. K., Ilaiyaraja, P., and Sudakar, C. (2018a). Whispering gallery mode assisted enhancement in the power conversion efficiency of DSSC and QDSSC devices using $\mathrm{TiO}_{2}$ microsphere photoanodes. ACS Appl. Energy Mater. 1, 765-774. doi: 10.1021/acsaem.7b00207

Das, T. K., Ilaiyaraja, P., and Sudakar, C. (2018b). Whispering gallery mode enabled efficiency enhancement: defect and size controlled CdSe quantum dot sensitized whisperonic solar cells. Sci. Rep. 8:9709. doi: 10.1038/s41598-018-27969-y

Debye, P. (1909). Der Lichtdruck auf Kugeln von beliebigem Material. Ann. Phys. 335, 57-136. doi: 10.1002/andp.19093351103

Dwivedi, C., Dutta, V., Chandiran, A. K., Nazeeruddin, M. K., and Grätzel, M. (2013). Anatase $\mathrm{TiO}_{2}^{-}$hollow microspheres fabricated by continuous spray pyrolysis as a scattering layer in dye-sensitised solar cells. Energy Proc. 33, 223-227. doi: 10.1016/j.egypro.2013.05.061

Foreman, M. R., Swaim, J. D., and Vollmer, F. (2015). Whispering gallery mode sensors. Adv. Opt. Photon. 7, 168-240. doi: 10.1364/AOP.7.000168

Garrett, C. G. B., Kaiser, W., and Bond, W. L. (1961). Stimulated emission into optical whispering modes of spheres. Phys. Rev. 124, 1807-1809. doi: 10.1103/PhysRev.124.1807

Grandidier, J. (2012). Simulations of solar cell absorption enhancement using resonant modes of a nanosphere array. J. Photon. Energy 2:024502. doi: 10.1117/1.Jpe.2.024502

Grandidier, J., Callahan, D. M., Munday, J. N., and Atwater, H. A. (2011). Light absorption enhancement in thin-film solar cells using whispering gallery modes in dielectric nanospheres. Adv. Mater. 23, 1272-1276. doi: 10.1002/adma.201004393

Grandidier, J., Callahan, D. M., Munday, J. N., and Atwater, H. A. (2012). Gallium arsenide solar cell absorption enhancement using whispering gallery modes of dielectric nanospheres. IEEE J. Photovolt. 2, 123-128. doi: 10.1109/JPHOTOV.2011.2180512

Grandidier, J., Weitekamp, R. A., Deceglie, M. G., Callahan, D. M., Battaglia, C., Bukowsky, C. R., et al. (2013). Solar cell efficiency enhancement via light trapping in printable resonant dielectric nanosphere arrays. Phys. Status Solidi A 210, 255-260. doi: 10.1002/pssa.201228690

Ha, D., Fang, Z., Hu, L., and Munday, J. N. (2014). Paper-based antireflection coatings for photovoltaics. Adv. Energy Mater. 4:1301804. doi: 10.1002/aenm.201301804

Ha, D., Yoon, Y., and Zhitenev, N. B. (2018). Nanoscale imaging of photocurrent enhancement by resonator array photovoltaic coatings. Nanotechnology 29:145401. doi: 10.1088/1361-6528/aaab0c

\section{AUTHOR CONTRIBUTIONS}

AC wrote and formatted the article. TD and PI have discussed and given input to the manuscript. CS wrote and communicated the manuscript.

\section{ACKNOWLEDGMENTS}

CS acknowledges the support by DST-SERI through grant no. DST/TM/SERI/2K11/113. PI acknowledges the NPDF fellowship from DST-SERB through grant no. PDF/2016/000461.

Hetsch, F., Xu, X., Wang, H., Kershaw, S. V., and Rogach, A. L. (2011). Semiconductor nanocrystal quantum dots as solar cell components and photosensitizers: material, charge transfer, and separation aspects of some device topologies. J. Phys. Chem. Lett. 2, 1879-1887. doi: 10.1021/jz200802j

Hua, B., Lin, Q., Zhang, Q., and Fan, Z. (2013). Efficient photon management with nanostructures for photovoltaics. Nanoscale 5, 6627-6640. doi: $10.1039 / \mathrm{c} 3 \mathrm{nr} 01152 \mathrm{f}$

Ilaiyaraja, P., Das, T. K., Mocherla, P. S. V., and Sudakar, C. (2019). Optical whispering gallery-enabled enhanced photovoltaic efficiency of CdS-CuInS 2 thin film-sensitized whisperonic solar cells. J. Phys. Chem. C 123, 1579-1586. doi: 10.1021/acs.jpcc.8b09292

Ilaiyaraja, P., Kumar Das, T., Mocherla, P. S. V., and Sudakar, C. (2017). Wellconnected microsphere-nanoparticulate $\mathrm{TiO}_{2}$ composites as high performance photoanode for dye sensitized solar cell. Solar Energy Mater. Solar Cells 169, 86-97. doi: 10.1016/j.solmat.2017.05.001

Ilaiyaraja, P., Rakesh, B., Das, T. K., Mocherla, P. S. V., and Sudakar, C. (2018). $\mathrm{CuInS}_{2}$ quantum dot sensitized solar cells with high $\mathrm{V}_{\mathrm{OC}} \approx 0.9 \mathrm{~V}$ achieved using microsphere-nanoparticulate $\mathrm{TiO}_{2}$ composite photoanode. Solar Energy Mater. Solar Cells 178, 208-222. doi: 10.1016/j.solmat.2018.01.018

Jara, D. H., Yoon, S. J., Stamplecoskie, K. G., and Kamat, P. V. (2014). Sizedependent photovoltaic performance of $\mathrm{CuInS}_{2}$ quantum dot-sensitized solar cells. Chem. Mater. 26, 7221-7228. doi: 10.1021/cm5040886

Jun, H. K., Careem, M. A., and Arof, A. K. (2013). Quantum dot-sensitized solar cells-perspective and recent developments: a review of Cd chalcogenide quantum dots as sensitizers. Renew. Sustain. Energy Rev. 22, 148-167. doi: 10.1016/j.rser.2013.01.030

Kamat, P. V. (2008). Quantum dot solar cells. Semiconductor nanocrystals as light harvesters. J. Phys. Chem. C 112, 18737-18753. doi: 10.1021/jp806791s

Kang, G., Park, H., Shin, D., Baek, S., Choi, M., Yu, D.-H., et al. (2013). Broadband light-trapping enhancement in an ultrathin film a-Si absorber using whispering gallery modes and guided wave modes with dielectric surfacetextured structures. Adv. Mater. 25, 2617-2623. doi: 10.1002/adma.201204596

Kim, H., and Yong, K. (2013). A highly efficient light capturing 2D (nanosheet)-1D (nanorod) combined hierarchical $\mathrm{ZnO}$ nanostructure for efficient quantum dot sensitized solar cells. Phys. Chem. Chem. Phys. 15, 2109-2116. doi: 10.1039/C2CP44045H

Liao, J.-Y., He, J.-W., Xu, H., Kuang, D.-B., and Su, C.-Y. (2012). Effect of $\mathrm{TiO}_{2}$ morphology on photovoltaic performance of dye-sensitized solar cells: nanoparticles, nanofibers, hierarchical spheres and ellipsoid spheres. J. Mater. Chem. 22, 7910-7918. doi: 10.1039/C2JM16148F

Ling, X., Zhou, S., Yuan, J., Shi, J., Qian, Y., Larson, B. W., et al. (2019). 14.1\% $\mathrm{CsPbI}_{3}$ perovskite quantum dot solar cells via cesium cation passivation. Adv. Energy Mater. 9:1900721. doi: 10.1002/aenm.201900721

Mavrokefalos, A., Han, S. E., Yerci, S., Branham, M. S., and Chen, G. (2012). Efficient light trapping in inverted nanopyramid thin crystalline silicon membranes for solar cell applications. Nano Lett. 12, 2792-2796. doi: $10.1021 / \mathrm{nl} 2045777$

Mie, G. (1908). Beiträge zur Optik trüber Medien, speziell kolloidaler Metallösungen. Ann. Phys. 330, 377-445. doi: 10.1002/andp.19083300302

Mihi, A., Bernechea, M., Kufer, D., and Konstantatos, G. (2013). Coupling resonant modes of embedded dielectric microspheres in solution-processed solar cells. Adv. Opt. Mater. 1, 139-143. doi: 10.1002/adom.201200015 
Mora-Seró, I., Giménez, S., Fabregat-Santiago, F., Gómez, R., Shen, Q., Toyoda, T., et al. (2009). Recombination in quantum dot sensitized solar cells. Acc. Chem. Res. 42, 1848-1857. doi: 10.1021/ar900134d

Nozik, A. J. (2002). Quantum dot solar cells. Phys. E 14, 115-120. doi: 10.1016/S1386-9477(02)00374-0

O’Regan, B., and Grätzel, M. (1991). A low-cost, high-efficiency solar cell based on dye-sensitized colloidal $\mathrm{TiO}_{2}$ films. Nature 353, 737-740. doi: $10.1038 / 353737 \mathrm{a} 0$

Pernik, D. R., Tvrdy, K., Radich, J. G., and Kamat, P. V. (2011). Tracking the adsorption and electron injection rates of CdSe quantum dots on $\mathrm{TiO}_{2}$ : linked versus direct attachment. J. Phys. Chem. C 115, 13511-13519. doi: $10.1021 /$ jp203055d

Prasad, M. B. R., and Pathan, H. M. (2016). Effect of photoanode surface coverage by a sensitizer on the photovoltaic performance of titania based CdS quantum dot sensitized solar cells. Nanotechnology 27:145402. doi: 10.1088/0957-4484/27/14/145402

Qadir, M. B., Sun, K. C., Sahito, I. A., Arbab, A. A., Choi, B. J., Yi, S. C., et al. (2015). Composite multi-functional over layer: a novel design to improve the photovoltaic performance of DSSC. Solar Energy Mater. Solar Cells 140, 141-149. doi: 10.1016/j.solmat.2015.04.011

Rayleigh, L. (1910). CXII. The problem of the whispering gallery. Lond. Edinb. Dublin Philos. Mag. J. Sci. 20, 1001-1004. doi: 10.1080/14786441008636993

Rayleigh, L. (1914). IX. Further applications of Bessel's functions of high order to the Whispering Gallery and allied problems. Lond. Edinb. Dublin Philos. Mag. J. Sci. 27, 100-109. doi: 10.1080/14786440108635067

Rühle, S., Shalom, M., and Zaban, A. (2010). Quantum-dot-sensitized solar cells. Chem. Soc. Rev. 11, 2290-2304. doi: 10.1002/cphc.201000069

Sha, W. E. I., Ren, X., Chen, L., and Choy, W. C. H. (2015). The efficiency limit of $\mathrm{CH}_{3} \mathrm{NH}_{3} \mathrm{PbI}_{3}$ perovskite solar cells. Appl. Phys. Lett. 106:221104. doi: $10.1063 / 1.4922150$

Sharma, D., Jha, R., and Kumar, S. (2016). Quantum dot sensitized solar cell: recent advances and future perspectives in photoanode. Solar Energy Mater. Solar Cells 155, 294-322. doi: 10.1016/j.solmat.2016.05.062

Sharma, S., Jain, K. K., and Sharma, A. (2015). Solar cells: in research and applications-a review. Mater. Sci. Appl. 06, 1145-1155. doi: $10.4236 / \mathrm{msa} .2015 .612113$

Shen, X., Jia, J., Lin, Y., and Zhou, X. (2015). Enhanced performance of CdTe quantum dot sensitized solar cell via anion exchanges. J. Power Sources 277, 215-221. doi: 10.1016/j.jpowsour.2014.12.022

Shopova, S. I., Farca, G., Rosenberger, A. T., Wickramanayake, W. M. S., and Kotov, N. A. (2004). Microsphere whispering-gallery-mode laser using HgTe quantum dots. Appl. Phys. Lett. 85, 6101-6103. doi: 10.1063/1.1841459

Tachan, Z., Hod, I., Shalom, M., Grinis, L., and Zaban, A. (2013). The importance of the $\mathrm{TiO}_{2}$ /quantum dots interface in the recombination processes of quantum dot sensitized solar cells. Phys. Chem. Chem. Phys. 15, 3841-3845. doi: $10.1039 / \mathrm{C} 3 \mathrm{CP} 44719 \mathrm{G}$

Tang, Z., Tress, W., and Inganäs, O. (2014). Light trapping in thin film organic solar cells. Mater. Today 17, 389-396. doi: 10.1016/j.mattod.2014.05.008

Tran, H. N., Nguyen, V. H., Nguyen, B. H., and Vu, D. L. (2016). Light trapping and plasmonic enhancement in silicon, dye-sensitized and titania solar cells. Adv. Nat. Sci. 7:013001. doi: 10.1088/2043-6262/7/1/013001

van de Hulst, H. C. (1946). Optics of Spherical Particles (Recherches astronomiques de l'Observatoire d'Utrecht, 11, pt.1) Amsterdam: Drukkerij J.F. Duwaer.

Veluthandath, A. V., and Bisht, P. B. (2015). Radiative rate modification in CdSe quantum dot-coated microcavity. J. Appl. Phys. 118:233102. doi: $10.1063 / 1.4937577$

Venkatakrishnarao, D., Mamonov, E. A., Murzina, T. V., and Chandrasekar, R. (2018). Advanced organic and polymer whispering-gallery-mode microresonators for enhanced nonlinear optical light. Adv. Opt. Mater. 6:1800343. doi: 10.1002/adom.201800343

Wang, W., Wu, S., Reinhardt, K., Lu, Y., and Chen, S. (2010). Broadband light absorption enhancement in thin-film silicon solar cells. Nano Lett. 10, 2012-2018. doi: 10.1021/nl904057p

Wang, Y., Li, M., Zhou, X., Li, P., Hu, X., and Song, Y. (2018a). High efficient perovskite whispering-gallery solar cells. Nano Energy 51, 556-562. doi: 10.1016/j.nanoen.2018.06.085
Wang, Y., Wang, P., Zhou, X., Li, C., Li, H., Hu, X., et al. (2018b). Diffractiongrated perovskite induced highly efficient solar cells through nanophotonic light trapping. Adv. Energy Mater. 8:1702960. doi: 10.1002/aenm.201702960

Wang, Z.-S., Kawauchi, H., Kashima, T., and Arakawa, H. (2004). Significant influence of $\mathrm{TiO}_{2}$ photoelectrode morphology on the energy conversion efficiency of N719 dye-sensitized solar cell. Coord. Chem. Rev. 248, 1381-1389. doi: 10.1016/j.ccr.2004.03.006

$\mathrm{Xu}, \mathrm{Y}$. , Gong, T., and Munday, J. N. (2015). The generalized Shockley-Queisser limit for nanostructured solar cells. Sci. Rep. 5:13536. doi: 10.1038/srep13536

Yang, W. S., Park, B.-W., Jung, E. H., Jeon, N. J., Kim, Y. C., Lee, D. U., et al. (2017). Iodide management in formamidinium-lead-halide-based perovskite layers for efficient solar cells. Science 356:1376. doi: 10.1126/science.aan2301

Yang, Z., Gao, P., Zhang, C., Li, X., and Ye, J. (2016). Scattering effect of the high-index dielectric nanospheres for high performance hydrogenated amorphous silicon thin-film solar cells. Sci. Rep. 6:30503. doi: 10.1038/srep 30503

Yao, Y., Yao, J., Narasimhan, V. K., Ruan, Z., Xie, C., Fan, S., et al. (2012). Broadband light management using low-Q whispering gallery modes in spherical nanoshells. Nat. Commun. 3:664. doi: 10.1038/ncomms1664

Yin, G., Manley, P., and Schmid, M. (2016). Light absorption enhancement for ultra-thin $\mathrm{Cu}\left(\mathrm{In}_{1-\mathrm{x}} \mathrm{Ga}_{\mathrm{x}}\right) \mathrm{Se}_{2}$ solar cells using closely packed 2-D $\mathrm{SiO}_{2}$ nanosphere arrays. Solar Energy Mater. Solar Cells 153, 124-130. doi: 10.1016/j.solmat.2016.04.012

Yin, J., Zang, Y., Yue, C., He, X., Li, J., Wu, Z., et al. (2013). Self-assembled hollow nanosphere arrays used as low Q whispering gallery mode resonators on thin film solar cells for light trapping. Phys. Chem. Chem. Phys. 15, 16874-16882. doi: $10.1039 / \mathrm{c} 3 \mathrm{cp} 53162 \mathrm{~g}$

Yu, W. W., Qu, L., Guo, W., and Peng, X. (2003). Experimental determination of the extinction coefficient of CdTe, CdSe, and CdS nanocrystals. Chem. Mater. 15, 2854-2860. doi: 10.1021/cm034081k

Zaban, A., Mićić, O. I., Gregg, B. A., and Nozik, A. J. (1998). Photosensitization of nanoporous $\mathrm{TiO}_{2}$ electrodes with InP quantum dots. Langmuir 14, 3153-3156. doi: 10.1021/la9713863

Zhao, F., Tang, G., Zhang, J., and Lin, Y. (2012). Improved performance of CdSe quantum dot-sensitized $\mathrm{TiO}_{2}$ thin film by surface treatment with $\mathrm{TiCl}_{4}$. Electrochim. Acta 62, 396-401. doi: 10.1016/j.electacta.2011.12.047

Zhao, J., Wang, A., Altermatt, P., and Green, M. A. (1995). Twenty-four percent efficient silicon solar cells with double layer antireflection coatings and reduced resistance loss. Appl. Phys. Lett. 66, 3636-3638. doi: 10.1063/1.114124

Zhi, Y., Manchee, C. P. K., Silverstone, J. W., Zhang, Z., and Meldrum, A. (2012). Refractometric sensing with silicon quantum dots coupled to a microsphere. Plasmonics 8, 71-78. doi: 10.1007/s11468-012-9423-8

Zhi, Y., Thiessen, T., and Meldrum, A. (2013). Silicon quantum dot coated microspheres for microfluidic refractive index sensing. J. Opt. Soc. Am. B 30, 51-56. doi: 10.1364/JOSAB.30.000051

Zhou, H., Chen, Q., Li, G., Luo, S., Song, T.-B., Duan, H.-S., et al. (2014). Interface engineering of highly efficient perovskite solar cells. Science 345:542. doi: 10.1126/science. 1254050

Zhu, J., Yu, Z., Burkhard, G. F., Hsu, C.-M., Connor, S. T., Xu, Y., et al. (2009). Optical absorption enhancement in amorphous silicon nanowire and nanocone arrays. Nano Lett. 9, 279-282. doi: 10.1021/nl802886y

Zhu, Z., Qiu, J., Yan, K., and Yang, S. (2013). Building high-efficiency CdS/CdSesensitized solar cells with a hierarchically branched double-layer architecture. ACS Appl. Mater. Interfaces 5, 4000-4005. doi: 10.1021/am400235g

Conflict of Interest: The authors declare that the research was conducted in the absence of any commercial or financial relationships that could be construed as a potential conflict of interest.

Copyright (c) 2019 Cholasettyhalli Dakshinamurthy, Das, Ilaiyaraja and Sudakar. This is an open-access article distributed under the terms of the Creative Commons Attribution License (CC BY). The use, distribution or reproduction in other forums is permitted, provided the original author(s) and the copyright owner(s) are credited and that the original publication in this journal is cited, in accordance with accepted academic practice. No use, distribution or reproduction is permitted which does not comply with these terms. 